\title{
Genome-Wide Identification of Metal Tolerance Genes in Potato (Solanum Tuberosum): Response to Two Heavy Metal Stress
}

Dandan Li ( $\sim$ gs.lidd19@gzu.edu.cn )

Guizhou University

Guandi He

Guizhou University

Weijun Tian

Guizhou University

Yun Huang

Guizhou University

Lulu Meng

Guizhou University

Yeqing $\mathrm{He}$

Guizhou University

Danxia Wu

Guizhou University

Yao Liu

Guizhou University

Tengbing He

Guizhou University

\section{Research Article}

Keywords: Metal tolerance proteins, Phylogeny analysis, Cadmium stress, Zinc stress, Expression analysis

Posted Date: February 9th, 2021

DOI: https://doi.org/10.21203/rs.3.rs-166067/v1

License: (c) (i) This work is licensed under a Creative Commons Attribution 4.0 International License. Read Full License 


\section{Abstract}

Metal tolerance proteins play an important role in the transport and tolerance of divalent heavy metals in plant species. Potatoes are an important food crop whose yields can be deeply affected by heavy metals. However, there is a lack of information concerning the members and function of the MTP gene family in Solanum tuberosum. In this study, we identified and screened 11 MTP genes in potatoes which we named as StMTP1 to StMTP11 based on their positions on the chromosomes. Phylogenetic analysis divided these 11 MTP genes into three subfamilies; Mn-MTP, Zn-MTP and Zn/Fe-MTP. HXXXD and DXXXD conserved motifs were found on or around the transmembrane domain II and transmembrane domain $\mathrm{V}$ of these proteins. The highly conserved histidine and aspartic acid residues may be related to the transport of metal ions. Quantitative real-time polymerase chain reaction (qRT-PCR) analysis showed that the expression levels of StMTP9 and StMTP10 in leaf tissues increased by around 24-fold following cadmium stress for 24 hours. We hypothesize that StMTP9 and StMTP10 respond to cadmium stress. StMTP11 showed the highest level of expression in stem tissues after 6 hours of zinc stress at more than 13 times the level of expression in controls indicating that StMTP11 is more sensitive to zinc stress. In summary, our results further the current understanding of the molecular mechanisms regulated by members of the MTP gene family in plant responses to heavy metal stress.

\section{Introduction}

Zinc $(\mathrm{Zn})$ is an essential micronutrient element for plant growth and development that participates in several molecular processes including auxin synthesis, enzyme activation and protein synthesis (Jan et al. 2017). However, excessive Zn can have negative effects on plants by impacting the metabolism of flavonoids, reduce energy metabolism and cause toxicity to plants (Zhang et al. 2017). Cadmium ion $\left(\mathrm{Cd}^{2+}\right)$ is a heavy metal ion that is not essential for plant growth and development and can cause damage to plants even at low concentrations (Li et al. 2019).

To survive, plants must respond quickly to heavy metal stresses to adapt to their growing conditions and minimize metabolic losses (Vishwakarma et al. 2019). Plants have gradually evolved to have specific mechanisms for the active uptake and transport of these metals that allow them to regulate the cellular concentrations of heavy metals through transporters (Paulsen and Saier 1997). The cation transporter family (CDF) consists of membrane-bound proteins that can maintain the cellular homeostasis of metal ions in plants. CDF proteins have a central role in the tolerance of plants to heavy metal stress. CDF proteins are common divalent cation transporters found in archaea, bacteria and eukaryotes that function to regulate metal homeostasis (Migocka et al. 2014). In plants, the CDF transporter responsible for the transportation and isolation of metals to the vacuole is called the metal tolerance proteins (MTPs). Evidence suggests that MTPs may be involved in other processes within plants such as anti-oxidative stress, signal transduction, the development of plant nodules and in regulating the entry of $\mathrm{Zn}$ into the endoplasmic reticulum (Bruinsma et al. 2002,Leon-Mediavilla et al. 2018,Ellis et al. 2004).

According to different substrate specificities, the MTP family is divided into three subfamilies, namely Mn-MTP, Zn-MTP and Zn/Fe-MTP (Montanini et al. 2007). These members usually contain amino and carboxyl cytoplasmic ends and 4-6 transmembrane domains (TMDs) that are highly conserved signatures sequences in transmembrane domain II (TMDII) and transmembrane domain V (TMDV) (Erbasol et al. 2013). The first MTP protein identified in plants was Arabidopsis thaliana MTP1 (AtMTP1) (van der Zaal et al. 1999). Arabidopsis overexpressing AtMTP1 has a strong resistance to high $\mathrm{Zn}$ exposures and can significantly increase root $\mathrm{Zn}^{2+}$ concentrations (van der Zaal et al. 1999). In the presence of excessive $\mathrm{Zn}$ and $\mathrm{Cd}$, the expression of Citrus sinensis MTP1 (CitMTP1), MTP3(CitMTP3) and MTP4(CitMTP4) is significantly up-regulated in roots or leaves (Fu et al. 2017). The overexpression of Oryza sativa MTP1(OsMTP1) in tobacco results in increased accumulation activity and growth rate, and reduces the levels of phytotoxicity caused by cadmium stress (Fu et al. 2017). Triticum urartu MTP1(TUMTP1) has been shown to maintain plant homeostasis by isolating excess $\mathrm{Zn}^{2+}$ and $\mathrm{Co}^{2+}$ in vacuoles (Wang et al. 2018). It has also been shown in turnips that zinc, copper, 
manganese and cadmium can all induce the expression of MTPS (Wang et al. 2018). The heterologous expression of tea plant CSMTP8.2 in Arabidopsis results in tolerance to $\mathrm{Mn}^{2+}$ (Zhang et al. 2020). In Populus trichocarpa, MTP8.1

(PtrMTP8.1), MTP9 (PtrMTP9) and MTP10.4 (PtrMTP10.4) can transport $\mathrm{Mn}^{2+}$ in yeast cells, whilst the MTP6 (PtrMTP6) can transport $\mathrm{Co}^{2+}, \mathrm{Fe}^{2+}$ and $\mathrm{Mn}^{2+}$ (Gao et al. 2020). Studies have also shown that OsMTP11 is a trans-Golgi localization transporter of manganese in rice that functions to reduce heavy metal toxicity by chelating Mn in vacuoles (Ma et al. 2018; Tsunemitsu et al. 2018). These data highlight the role of MTPs in response to heavy metal stress.

Potatoes belong to the Solanaceae family and are a nutritious food stable that is one of the four important food crops in the world along with wheat, rice and corn. Currently, heavy metals are the main pollutants that affect the environment and soil quality of agricultural land. Potatoes that are planted in soil become contaminated by heavy metals and suffer from serious leaf deformities and limited growth and development that results in significantly decrease crop yields.

The MTP family is a heavy metal transporter that plays an important role in the response of plants to heavy metal stress. Current studies on MTPs have mainly focused on Arabidopsis, rice, tobacco and other plants with few reports in Solanum tuberosum. In this study, we identified 11 StMTPs and compared their physical and chemical properties with MTP genes in Arabidopsis, rice and tomatoes. The basic physical and chemical properties of StMTP proteins were characterized. Also, we analyzed the modulation of StMTP proteins in response to cadmium and zinc stress. This study provides an important understanding of the structure and function of StMTPs in Solanum tuberosum.

\section{Materials And Methods}

\section{Identification of MTP gene family members and chromosome locations}

The genome annotation files and protein files of Solanum tuberosum, Solanum_lycopersicum, Arabidopsis thaliana were downloaded from the ensemble database (http://plants.ensembl.org/index.html). Then downloaded the hidden Markov model matrix file of the conservative domains of MTP family genes in the pfm database (http://pfam.xfam.org/family/PF01545). The HMM search program of HMMER (ver3.1) in the BioLinux system was used to retrieve information from the protein sequence database in potatoes and set an E-value set to $1.2 \mathrm{e}-28$ to obtain the candidate MTP domain sequence. SMART (http://smart.embl.de/), NCBI CDD

(https://doi.org/www.ncbi.nlm.nih.gov/cdd/) and pfam (http://pfam.xfam.org/) were used to confirm the domains of the MTP genes in potato, tomato, and Arabidopsis. The identified MTP family protein sequences in rice were downloaded from phytozome (https://doi.org/phytozome.jgi.doe.gov/pz/portal.html). After obtaining the location information of the genes, we used the online software MapGene2Chrom web v2 (http://mg2c.iask.in/mg2c_v2.0/) to draw a gene chromosome location map. Multiple Collinearity Scan (MCScan) analysis was used to determine the homology between horse Solanum tuberosum, Solanum lycopersicum, Arabidopsis thaliana and Oryza sativa of the MTP genome (Wang et al. 2012).

\section{Phylogenetic analysis of the potato MTP gene family}

The protein sequences were introduced into MEGA-X (v10.1.8) and the clustalw in MEGA-X program (v10.1.8) used for comparison. The aligned files were used to construct a phylogenetic tree of the family members of potato, tomato, Arabidopsis, tobacco and rice using the neighbor-joining method. The execution parameters were No. Of differences mode, $30 \%$ deletion, and 1000 repeats (Khan et al. 2019). The constructed evolutionary tree was then imported into the Evolview (https://doi.org/evolgenius.info//evolview-v2/\#login) online software for presentation.

\section{Analysis of gene structures and conserved domains in potato and Arabidopsis}


Obtained the position information of the MTP gene exons, CDS and UTR of potato and Arabidopsis through the bio-linux system and imported the data into Tbtools v1.0692 for mapping. The potato and Arabidopsis protein sequences were imported into the meme software for motif analysis and the results were imported into TBtools 1.0692 for mapping. The data was combined with the gene structure map and conserved motif map using Adobe Illustrator CC 2019 software.

\section{Physicochemical analysis of potato MTP family genes}

Using the online analysis tool ExPASyProtParam (https://web.expasy.org/protparam/), the physical and chemical properties of the selected potato MTP genes were analyzed (Li et al. 2019). Plant-mPLoc

(http://www.csbio.sjtu.edu.cn/bioinf/plant -multi/) was used to predict the subcellular locations of proteins and the TMHMM Server v. 2.0 was used to predict the domains of the StMTP family members.

\section{Amino acid sequences of the potato MTP}

Potato and Arabidopsis proteins were imported into clustalx-2.0.11 for comparison and the results were then imported into the GENEDOC software for further analysis. Protein transmembrane region predictions were made using novopro (https:// www.novopro.cn/tools/tmhmm.html).

\section{Promoter analysis}

The 2,000 bp sequence upstream of the transcription start site of the potato MTP gene family members was extracted and Plant CARE (http://bioinformatics.psb.ugent.be/webtools/plantcare/html/) used to predict the promoter cis-acting elements. Origin 2018 64Bit was used to produce images of the gene promoters.

\section{Experimental materials and heavy metal stress}

In this experiment, Yunshu 505 (Solanum tuberosum) was used as the experimental model and was planted in pots at the experimental base of Guizhou University in June 2020. 100 mg/kg CdCl2 (Analytical grade, Tianjin Kemil Chemical Reagent Co., Ltd.) and 100 mg/kg ZnCl2 (Analytical grade, Chengdu Gold Reagent Co., Ltd.) were used to induce stress during the flowering period of potatoes (Tian et al. 2021).

After 6,12 and $24 \mathrm{~h}$ of heavy metal stress, the middle leaves, roots $(2 \mathrm{~g})$ and stems $(5 \mathrm{~g})$ were taken from the potato plants, placed in liquid nitrogen and stored in the refrigerator at $-80^{\circ} \mathrm{C}$. Total RNA was isolated from the potato roots, stems and leaves using a Total RNA Extraction Reagent (Vazyme) reagent. cDNA was synthesized using a StarScript II First-strand cDNA Synthesis Mix with a Gdna Remover kit. The internal reference and target gene sequences were simultaneously amplified using the cDNA as a template. Each sample was amplified 3 times. Gene expression was measure by CFX96 Real-time PCR System (BIO-RAD CFX96, USA). A GenStar (Beijing Kangrun Chengye Biotechnology Co., Ltd.) and 2RealStar Green Fast Mixture PCR kit was used in the experiment comprising a $20 \mu$ l reaction system of the following reagents: $10 \mu \mathrm{L}$ of $2 \times$ RealStar Green Fast Mixture, $1 \mu \mathrm{L}$ of primers, $7 \mu \mathrm{L}$ of RNase-Free ddH2O and $2 \mu \mathrm{L}$ of cDNA. A two-step protocol was used that consisted of $95^{\circ} \mathrm{C}$ for $3 \mathrm{~min}, 95^{\circ} \mathrm{C}$ for $15 \mathrm{~s}$ and $65^{\circ} \mathrm{C}$ for $15 \mathrm{~s}$ for a total of 40 reaction cycles. The $2^{-\Delta \Delta C t}$ algorithm was used for data analysis.

\section{Results And Analysis}

\section{Chromosome locations and collinearity analysis}

Eleven MTP genes were identified from potato and defined as StMTP1-StMTP11 according to their chromosomal positions (Table S1). Chromosome mapping was performed online (http://mg2c.iask.in/mg2c_v2.0/) and the results presented in Fig. 1 shows that the MTP genes of potato are unevenly distributed on the 12 chromosomes of potato and 
are mainly located on 8 chromosomes. Most genes are found on chromosomes 3, 7 and 12 and one chromosome has 2 MTP genes. StMTP2 and StMTP4 are located in the middle of a chromosome and the remaining 9 StMTP genes are located at both ends of a chromosome.

To further investigate the evolutionary mechanism of the StMTP genes, the potato and the model plants (Arabidopsis, rice) and the Solanaceae plant tomato were synonymously compared to identify the orthologous MTP gene (Fig. 2). A total of 12 collinearity gene pairs were found as follows (Table S2); StMTP5:AtMTP11, StMTP6:AtMTP4, StMTP5:0s01t0837800-01, StMTP2:SIMTP2, StMTP3:SIMTP3, StMTP4:SIMTP4, StMTP5:SIMTP5, StMTP6:SIMTP6, StMTP7:SIMTP7, StMTP9:SIMTP8, StMTP10:SIMTP9 and StMTP11:SIMTP10.

From the above results, the most collinearity gene pairs (9 pairs) were found in potato and tomato indicating high levels of genetic similarity. We found that the potato StMTP 5 gene has a continuous collinearity gene pair in rice, Arabidopsis and tomato.

\section{Identification, phylogeny and classification of potato MTP genes}

To investigate the evolution of the StMTP and the classification of its subfamily, we used the reported Arabidopsis (12) and rice (10) MTP genes and the identified tomato (10) and potato (11) MTP protein sequences(indentified by our laboratory to construct an evolutionary tree (Fig. 3). Based on previous studies, the MTP family can be divided into 7 different groups (groups 5, 12, 1, 6, 7, 9, 8) and 3 sub-families (Mn-MTP, Zn/Fe-MTP, Zn-MTP) (Montanini et al. 2007). Each subfamily has a different number of StMTPs. Mn-MTP contains 6 StMTPs and Zn-MTP contains 5, whilst the Zn/Fe-MTP subfamily does not contain any StMTPs.

\section{Gene structure and motif analysis}

Exons and introns in the structure of genes play an important role in the analysis of gene family evolution. Structural analysis of the MTP gene family (Fig. 4A) showed that groups 12 and 1 had fewer introns and only contained one CDS region. Group 12 did not contain introns. In group 1, 29\% of StMTP genes did not contain any introns whilst 71\% contained an intron. The remaining groups contained more introns ranging from 3-12. Of these, groups 4 and 5 contain the most introns with 11 and 12 introns, respectively. In general, the intron length in potatoes is longer than that of Arabidopsis. Also, five potato MTP genes (StMTP4, StMTP2, StMTP10, StMTP11, StMTP3) were also found to have no UTR structure.

We used the MEME online software to analyze the motif types and sequences of MTP gene family members in Arabidopsis and potatoes and found 20 motifs. In the same group, the number and types of motifs were mostly the same indicating conservation of the MTP structure. Differences were observed in the number of conserved motifs in the Zn-MTP subfamily (groups 5, 12 and 1) and the StMTP4 in group 5 lacked motif 14. All of the Zn-MTP subfamily members contained motifs 6 and 7. Each group contained several unique motifs. For example, motif 19 was found only in group 2 and group 3 only contained motifs 4, 12 and motif 9.The MTP signature sequence (Table S5) was found in motifs 6 (SLALLTDAAHLLSD) and 12 (SHGVTSDSAHSHDD) (Desbrosses-Fonrouge et al., 2005)

\section{StMTPs protein characteristics and prediction of subcellular localization}

We used online software to predict the characteristics of proteins encoded by the MTP family genes of potatoes and the results are summarized in Table 1. The molecular weight of the proteins encoded by these genes ranged from 28.77$100.11 \mathrm{kDa}$ and the isoelectric points varied from 5.01-8.96. The protein instability index can be used to determine the stability proteins with values $>40$ indicating unstable proteins. From our analysis, $73 \%$ of the proteins encoded by the StMTP gene were unstable and $27 \%$ were stable. Hydrophilicity predictions showed that $73 \%($ GAVY $>0)$ of the proteins were hydrophobic. Subcellular localization analysis found most of the proteins were located in the vacuole. However, 
StMTP9 and StMTP10 existed in both the vacuole and the cell membrane. Domain prediction found that StMTP2 had 12 transmembrane domains and StMTP4 had 1 transmembrane domain. The other StMTPs all contained 4-6 transmembrane domains.

Table 1

The information of MTP gene family in potato

\begin{tabular}{|c|c|c|c|c|c|c|c|}
\hline $\begin{array}{l}\text { Gene } \\
\text { Name }\end{array}$ & Accession No. & $\begin{array}{l}\text { MW } \\
\text { (KDa) }\end{array}$ & PI & $\begin{array}{l}\text { Instability } \\
\text { index (II) }\end{array}$ & GRAVY & $\begin{array}{l}\text { Subcellular } \\
\text { localization }\end{array}$ & $\begin{array}{l}\text { TMD } \\
\text { number }\end{array}$ \\
\hline StMTP1 & PGSC0003DMT400081988 & 45.43 & 5.07 & 43.26 & 0.03 & Vacuole. & 5 \\
\hline StMTP2 & PGSC0003DMT400017877 & 100.11 & 6.97 & 42.2 & -0.04 & Vacuole. & 12 \\
\hline StMTP3 & PGSC0003DMT400002845 & 44.83 & 5.01 & 48.32 & 0.12 & Vacuole. & 4 \\
\hline StMTP4 & PGSC0003DMT400038744 & 28.77 & 7.17 & 46.81 & -0.21 & Vacuole. & 1 \\
\hline StMTP5 & PGSC0003DMT400060459 & 38.96 & 5.22 & 44.16 & 0.24 & Vacuole. & 4 \\
\hline StMTP6 & PGSC0003DMT400077991 & 42.50 & 5.85 & 28.8 & 0.14 & Vacuole. & 6 \\
\hline StMTP8 & PGSC0003DMT400078996 & 39.47 & 5.97 & 34.53 & 0.19 & Vacuole. & 6 \\
\hline StMTP9 & PGSC0003DMT400029243 & 51.40 & 6.63 & 46.16 & 0.03 & $\begin{array}{l}\text { Cell } \\
\text { membrane.Vacuole. }\end{array}$ & 6 \\
\hline StMTP10 & PGSC0003DMT400024996 & 40.00 & 8.96 & 42.9 & -0.02 & $\begin{array}{l}\text { Cell } \\
\text { membrane.Vacuole. }\end{array}$ & 4 \\
\hline StMTP11 & PGSC0003DMT400010945 & 46.38 & 5.35 & 46.56 & 0.03 & Vacuole. & 4 \\
\hline
\end{tabular}

\section{Amino acid sequence alignment of StMTPs subfamily}

From Fig. 5 it can be seen that the protein sequence of StMTP is similar to Arabidopsis and rice (60\%-100). In this study, aspartic acid-based DXXXD (X = any amino acid) motifs onTMDIl and near TMDV were found in the Mn-MTP. HXXXD motifs ( $X$ = any amino acid) containing aspartic $(D)$ and histidine $(H)$ were found on TMD2 and TMD5 of Zn-MTP and $\mathrm{Zn} /$ Fe-MTP. There is a histidine-rich regions between the Zn-MTP subfamily transmembrane domain IV (TMDIV) and the TMDV. Also, compared to the Zn-MTP and Zn/Fe-MTP subfamilies, the amino acids of several species in Mn-MTP are more similar indicating that this subfamily is more highly conserved than the other two subfamilies.

\section{Analysis of homeopathic components}

The promoter sequences of the 2,000 bp upstream region of the start codon in the StMTP1-11 genes were obtained and analyzed by Plantcare. The analysis showed 46 different types of action elements amongst which there were 18 types of photo-responsive components (such as GATA-motif, ACE, AE-box, Box-4, G-box and MRE). Homeopathic elements that respond to hormones (auxin, salicylic acid, abscisic acid and methyl jasmonate) include the AuxRR-core, SARE, the TCAelement, and the ABRE and the CGTCA-motif. In response to stress signals (low temperature, drought, and anaerobic induction) LTR, MBS, ARE and cis-acting elements are involved in defense and stress response TC-rich repeats. Also, some uncommon functional elements were found such as the CAT-box that relates to meristem expression, the 02-site which regulates Zn metabolism, HD-Zip1 that is related to the differentiation of palisade mesophyll cells and the cisregulatory RY-element that is involved in seed-specific regulation.

From Fig. 6 it can be seen that each member contained a large number of promoter core elements such as the CAAT- and the TATA-boxes. Also, each member contained more light-responsive elements. Each member contained cis-acting elements related to growth hormones such as abscisic acid, auxin, and salicylic acid. Amongst these genes, StMTP3 and 
StMTP8 had cis-acting regulatory elements involved in seed-specific regulation and $73 \%$ of the MTP genes contained cis-acting regulatory elements required for anaerobic induction.

\section{The expression patterns of StMTP in different tissues under heavy metal stress}

Real-time quantitative qRT-PCR was used to analyze the expression of StMTPs under heavy metal stress. 10 StMTP genes were selected for $\mathrm{Cd}^{2+}$ and $\mathrm{Zn}^{2+}$ stress and the expression patterns were determined in different tissues and at different times. As shown in Fig. 7, the expression of StMTPs in root tissues decreased compared to controls(0h), while the expression of StMTPs in leaf tissues significantly increased. These data show that the response of StMTPs in leaves is faster than in roots following heavy metal stress during the flowering period. In root tissues under $100 \mathrm{mg} / \mathrm{kg}$ of cadmium stress, StMTP3 was up-regulated 3.61 times at $6 \mathrm{~h}$ and StMTP1 was up-regulated by 2.55 times at $12 \mathrm{~h}$. The other genes in the roots were downregulated or not significantly upregulated. In stem tissues, except for StMTP1, the other genes were down-regulated at $12 \mathrm{~h}$. Amongst the downregulated genes, StMTP11 was most prominent. In leaf tissues, the relative expression levels of all genes were the highest at $24 \mathrm{~h}$, amongst which StMTP9 and StMTP10 were both up-regulated around 24 fold.

With $100 \mathrm{mg} / \mathrm{kg}$ zinc-stressed, the expression of StMTP3 in the root tissue reached the highest value after 6 hours which around 8 times that of the control. The other genes showed no significant expression changes in the roots. In stem tissues, the expression level of StMTP11 was the highest at $6 \mathrm{~h}$, reaching 13.59 times that of the control. After $12 \mathrm{~h}$ of stress, except for StMTP1, the rest of the genes showed a downward-regulated trend.After 6 hours of treatment in the leaves, all StMTPs were down-regulated.Except for StMTP1 and StMTP6, which reached the highest expression level at $24 \mathrm{~h}$, the expression levels of other StMTPs were all significantly up-regulated at $12 \mathrm{~h}$. For example, StMTP3 and StMTP10 were 5.23 times and 4.56 times the control. 
Table 2

Primers for StMTP gene expression analysis

\begin{tabular}{|c|c|c|}
\hline Primer name & Primer sequence & Purification method \\
\hline StMTP1 R & TGATCCCTACTGGCTGCACTCG & PAGE \\
\hline StMTP1 F & GATCTCATGGCTGGTGGCATACTG & PAGE \\
\hline StMTP2 R & GAGCAGGCAGGATCAGCAACAAG & PAGE \\
\hline StMTP2 F & ACCACCAACACCGCCACATTG & PAGE \\
\hline StMTP3 R & AAAATTCAGGAGGGGCGGATTGTC & PAGE \\
\hline StMTP3F & TGGCTGCTGTCCTCGGTGATC & PAGE \\
\hline StMTP4 R & ACACAACTCGGCGGTAGAATAAGC & PAGE \\
\hline StMTP4 F & AGGGGTTCCTCGTCTCTGTCATC & PAGE \\
\hline StMTP6 F & CCACAGCCTCGCGGTTCTAAC & PAGE \\
\hline StMTP7 R & CCATGTCAGCATCGGCATCAGG & PAGE \\
\hline StMTP7 F & TTTGCGAGATGGAGGAGGTTGTTG & PAGE \\
\hline StMTP8 R & ATCCAGCACCATATCAGCATCAGC & PAGE \\
\hline StMTP8 F & GGCTTGAGAAGGGGCTTTGTGAG & PAGE \\
\hline StMTP9 R & AGGAGACGACGGACACTAAAGGAG & PAGE \\
\hline StMTP9 F & GCTGCTCAGGCGGTAGTTGATC & PAGE \\
\hline StMTP10 R & GAGCGGTGTTGAGGAAGATGGAAG & PAGE \\
\hline StMTP10 F & TGGTGGTAGTGGTGGTGGTGATAG & PAGE \\
\hline StMTP11 R & GATTCCAACGGGCTGCACTCTC & PAGE \\
\hline StMTP11 F & CCTCATGGCTGGTGGCATACTATG & PAGE \\
\hline ActinF & AGCCACCACTGAGCACAATGTTAC & PAGE \\
\hline ActinR & AGAGGTTCCGTTGCCCAGAGG & PAG \\
\hline
\end{tabular}

\section{Discussion}

Transmembrane transport plays a key role in controlling the distribution of metals between organs and compartments in cells. The transport proteins in plants mainly include heavy metal ATPase (HMA) (He et al. 2020), natural resistanceassociated macrophage protein (NRAMP) (Lu et al. 2020), ATP-Bing Cassette (ABC) and the efflux proteins in the cationic diffusion promoter family, which is also called metal tolerance proteins. Metal cation binds to the cytoplasmic C-terminal domain (CTD) of the CDF protein causing the apolipoprotein to change from an open V-type dimer to a closed structure. The transmembrane domain then undergoes a conformational change allowing the transport of the metal cation (Barber-Zucker et al. 2019). Currently, the identification and functional analysis of MTP family members have been completed in grapes (Shirazi et al. 2019), rice (Farthing et al. 2017), tobacco (Liu et al. 2019) and other species but they are yet to be reported in potatoes. 
Plant MTP genes are divided into 7 groups (Cotrim et al. 2019). The first branch contains MTP1-MTP4 (G1), MTP5 (G5) and MTP12 (G12) which belong to the Zn-MTP subfamily. The second branch is composed of MTP7 (G7) and MTP6 (G6) which are derived from Zn/Fe-MTP. The third branch consists of MTP8 (G8) and MTP9-MTP11 (G9) from the MnMTP subfamily. In the Zn-MTP subfamily, MTP1 is related to the transport of $\mathrm{Zn}^{2+}, \mathrm{Cd}^{2+}, \mathrm{Ni}^{2+}$ and $\mathrm{Co}^{2+}(\mathrm{Chen}$ et al. 2009; Kim et al. 2004). The MTP2 protein mediates the transport of Zn, Co and Ni (Papierniak-Wygladala et al. 2020) and MTP3 mediates $\mathrm{Zn}$ and Mn tolerance by chelating the metal ions into the vacuoles (Gu et al. 2020). The CsMTP5CSMTP12 complex in cucumber is involved in the outflow of zinc from the cytoplasm of yeast cells (Migocka et al. 2018). The members of the Mn-MTP subfamily are mainly involved in the transport of manganese in plants (Chen et al. 2013; Ueno et al. 2015). There are currently few reports on the functional roles of members of the Zn/Fe-MTP subfamily. These reports indicate that the MTP gene is involved in the transport and tolerance to multiple heavy metals and plays an important role in maintaining metal homeostasis in plants.

In this study, 11 potato MTP genes were identified and screened. A phylogenetic tree was constructed and used to compare the identified potato MTP protein sequences with the phylogenetic distribution of the MTP sequences of known species such as rice and Arabidopsis thaliana. This may help to understand the function of potato MTP.

We found that the Zn/Fe-MTP subfamily did not contain any StMTPs and so we hypothesize that this subfamily had been lost during the evolution of the potato. Most potato and tomato genes were located in adjacent branches the species are closely related. The length of the proteins encoded by StMTPs ranged from 28.77-100.11 kDa. The predicted results of the subcellular localization indicated that all StMTPs are located in the vacuole which agreed with the results of Gao (2020). The predicted results of the TMHMM Server v. 2.0 analysis showed that StMTP4 has a transmembrane domain that is similar to OsMTP6 (without the domain) (Ram et al. 2019). StMTP2 has 12 transmembrane domains similar to 14 in Brassica rapa var. rapa MTP12 (BrrMTP12) (Li et al. 2018). Also Montanini (2007b) found that in two subfamilies of Zn-MTP and Zn/Fe-MTP, the highly conserved HXXXD (X = any amino acid) motifs of aspartic (D) and histidine $(H)$ were identified near the TMDII and TMDV. The Mn-MTP sequence can be distinguished by the consensus sequence $\operatorname{DxxxD}(\mathrm{x}=$ any amino acid) in the TMD $\mathrm{V}$, where $\mathrm{D}$ is a highly conserved aspartic acid residue (Montanini et al. 2007).

Divalent d-block metal cations (DDMC) such as $\mathrm{Zn}^{2+}, \mathrm{Mn}^{2+}, \mathrm{Fe}^{2+}$ and $\mathrm{Cu}^{2+}$ can interact with negatively charged (Asp, Glu) and polar residue (such as Cys, His and Asn) containing protein to reduce their toxicity to plants (Montanini et al. 2007). In this study, aspartic acid-based DXXXD motifs were found on the TMDII or near the TMDV of Mn-MTP and the HXXXD motifs on Zn-MTP, Zn/Fe-MTP TMDII and TMDV. Studies have shown that the highly conserved histidine and aspartic acid residues may be related to the transport of metal ions (Russell and Soulimane 2012). Also, a histidine-rich region between the Zn-MTP subfamily TMDIV and TMDV was observed that is responsible for zinc-binding (Migeon et al. 2010).

A gene promoter is a DNA sequence located upstream of the coding region of a gene and contains multiple cis-acting elements that are specific binding sites for proteins involved in transcription initiation and regulation (Hernandez-Garcia and Finer 2014). Promoter prediction analysis found a large number of promoter core elements such as the CAAT-box, TATA-box and light-responsive elements. Each member contained cis-acting elements related to growth hormones such as abscisic acid, auxin, and salicylic acid. We hypothesize that these genes may be regulated by plant hormones.

We studied the relative expression of 11 potato MTP genes under heavy metal stress $\left(\mathrm{Zn}^{2+}, \mathrm{Cd}^{2+}\right)$. Our results showed that these two metal ions can induce the expression of potato MTP genes indicating that potato MTP genes may be involved in the tolerance or transport of $\mathrm{Zn}^{2+}$ and $\mathrm{Cd}^{2+}$. Also, the overall expression levels of the MTP genes in leaves were higher than in roots and stems. Under cadmium stress, the relative expression levels of all genes in the leaves highest at $24 \mathrm{~h}$. Of these genes, STMTP9 and StMTP10 were both upregulated around 24 fold at $24 \mathrm{~h}$ indicating that 
these genes are involved in response to cadmium stress. Following zinc treatment, except for StMTP1 and StMTP6

which reached the highest expression level at $24 \mathrm{~h}$, the rest of StMTPs reached the highest expression levels at $12 \mathrm{~h}$ and all StMTPs were all down-regulated at $6 \mathrm{~h}$. The expression of StMTP11 was highest after 6 hours of zinc stress reaching 13 times that of the control suggesting that StMTP11 is more sensitive to zinc stress.

Overall, this study identified and analyzed the MTP genes in potatoes to provide further understanding of the structure and function of these genes. However, further studies are needed to better understand the response mechanism of StMTPs to heavy metal stress.

\section{Conclusions}

We explored the characteristics of the members of the StMTP family genes in potato and their expression patterns in different parts of the plant in response to heavy metal stresses. Phylogenetic analysis showed that the Zn/Fe-MTP subfamily was lost in potato evolution. By comparing the amino acid sequences of the StMTP subfamily, we showed that Zn-MTP and Zn/Fe-MTP had the HXXXD motif and the consensus sequence DxxxD in Mn-MTP. These two amino acid motifs may be related to the transport of metal ions. A region related to zinc transport was also found in Zn-MTP. Using qRT-PCR analysis, we found StMTP9 and StMTP10 are related to cadmium stress, and StMTP11 is more sensitive to zinc stress. Our results are partially consistent with previous studies and can provide important genetic data on the role of MTP members in potatoes.

\section{Declarations}

\section{Author's Contributions}

TBH and DDL conceived the idea of experiments.DDL, GDH, WJT, YH, LLM, YQH, DXW and YL carried out the experiments. DDL, YH, GDH and WJT analyzed the data, DD L, GDH and WJT wrote the manuscript. All authors read and approved the final manuscript.

\section{Acknowledgments}

All the authors thank the raw data download from the public database. We are very grateful to the editor and reviewers for critically evaluating the manuscript and providing constructive comments for its improvement.

\section{Funding}

This work was supported by the Program Foundation of Institute for Scientific Research of Karst Area of NSFC-GZGOV (U1612442), the Guizhou Science and Technology Agency Fund for Basic-condition Platform (2019-5701), as well as the Construction Program of Biology First-class Discipline in Guizhou (GNYL2017009).

\section{Data availability}

The datasets used and/or analyzed during the current study are available from the corresponding author on reasonable request.

\section{Ethics approval and consent to participate}

Not applicable.

\section{Consent for publication}


Not applicable.

\section{Conflicts of interest}

The authors declare that they have no competing interests.

\section{References}

1. Barber-Zucker S et al. (2019) Metal binding to the dynamic cytoplasmic domain of the cation diffusion facilitator (CDF) protein MamM induces a 'locked-in' configuration The FEBS Journal. 286:2193-2215. https://doi.org/10.1111/febs.14795

2. Barber-Zucker S, Shaanan B, Zarivach R (2017) Transition metal binding selectivity in proteins and its correlation with the phylogenomic classification of the cation diffusion facilitator protein family. Sci Rep-Uk. 7:16381. https://doi.org/10.1038/s41598-017-16777-5

3. Bruinsma JJ, Jirakulaporn T, Muslin AJ, Kornfeld K (2002) Zinc lons and Cation Diffusion Facilitator Proteins Regulate Ras-Mediated Signaling. Dev Cell 2:567-578. https://doi.org/10.1016/S1534-5807(02)00151-X

4. Chen M, Shen X, Li D, Ma L, Dong J, Wang T (2009) Identification and characterization of MtMTP1, a Zn transporter of CDF family, in the Medicago truncatula. Plant Physiol Bioch 47:1089-1094.

https://doi.org/10.1016/j.plaphy.2009.08.006

5. Chen $\mathrm{Z}$ et al. (2013) Mn tolerance in rice is mediated by MTP8.1, a member of the cation diffusion facilitator family. J Exp Bot 64:4375-4387. https://doi.org/10.1093/jxb/ert243

6. Cotrim CA, Jarrott RJ, Martin JL, Drew D (2019) A structural overview of the zinc transporters in the cation diffusion facilitator family Acta crystallographica. Section D, Structural biology 75:357-367. https://doi.org/doi.org/10.1107/S2059798319003814

7. Das N, Bhattacharya S, Maiti MK (2016) Enhanced cadmium accumulation and tolerance in transgenic tobacco overexpressing rice metal tolerance protein gene OsMTP1 is promising for phytoremediation .Plant Physiol Bioch 105:297-309. https://doi.org/10.1016/j.plaphy.2016.04.049

8. Ellis CD, Wang F, MacDiarmid CW, Clark S, Lyons T, Eide DJ (2004) Zinc and the Msc2 zinc transporter protein are required for endoplasmic reticulum function. The Journal of cell biology 166:325-335. https://doi.org/10.1083/jcb.200401157

9. Erbasol I, Bozdag GO, Koc A, Pedas P, Karakaya HC (2013) Characterization of two genes encoding metal tolerance proteins from Beta vulgaris subspecies maritima that confers manganese tolerance in yeast. Biometals 26:795-804. https://doi.org/10.1007/s10534-013-9658-7

10. Farthing EC, Menguer PK, Fett JP, Williams LE (2017) OsMTP11 is localised at the Golgi and contributes to Mn tolerance. Sci Rep-Uk. 7:15258. https://doi.org/10.1038/s41598-017-15324-6

11. Fu X et al. (2017) Genome-wide identification of sweet orange (Citrus sinensis) metal tolerance proteins and analysis of their expression patterns under zinc, manganese, copper, and cadmium toxicity. Gene 629:1-8. https://doi.org/10.1016/j.gene.2017.07.072

12. Gao Y et al. (2020) Genome-Wide Identification of Metal Tolerance Protein Genes in Populus trichocarpa and Their Roles in Response to Various Heavy Metal Stresses. Int J Mol Sci 21:1680. https://doi.org/10.3390/ijms21051680

13. Gu D et al. (2020) Expression of a Brassica napus metal transport protein (BnMTP3) in Arabidopsis thaliana confers tolerance to Zn and Mn. Plant Sci 110754. https://doi.org/ 10.1016/j.plantsci.2020.110754

14. He G, Qin L, Tian W, Meng L, He T, Zhao D (2020) Heavy Metal Transporters-Associated Proteins in S. tuberosum: Genome-Wide Identification, Comprehensive Gene Feature, Evolution and Expression Analysis. Genes-Basel 
11:1269. https://doi.org/10.3390/genes11111269

15. Hernandez-Garcia CM, Finer JJ (2014) Identification and validation of promoters and cis-acting regulatory elements. Plant Sci 217-218:109-119. https://doi.org/ 10.1016/j.plantsci.2013.12.007

16. Jan AU, Hadi F, Midrarullah, Nawaz MA, Rahman K (2017) Potassium and zinc increase tolerance to salt stress in wheat (Triticum aestivum L.). Plant Physiol Bioch 116:139-149. https://doi.org10.1016/j.plaphy.2017.05.008

17. Khan IU et al. (2019) Identification of novel rice (Oryza sativa) HPP and HIPP genes tolerant to heavy metal toxicity .Ecotox Environ Safe 175:8-18. https://doi.org/ 10.1016/j.ecoenv.2019.03.040

18. Kim D, Gustin JL, Lahner B, Persans MW, Baek D, Yun D, Salt DE (2004) The plant CDF family member TgMTP1 from the $\mathrm{Ni} / \mathrm{Zn}$ hyperaccumulator Thlaspi goesingense acts to enhance efflux of $\mathrm{Zn}$ at the plasma membrane when expressed in Saccharomyces cerevisiae. The Plant Journal 39:237-251. https://doi.org/10.1111/j.1365313X.2004.02126.X

19. Kobae Y, Uemura T, Sato MH, Ohnishi M, Mimura T, Nakagawa T, Maeshima M (2004) Zinc Transporter of Arabidopsis thaliana AtMTP1 is Localized to Vacuolar Membranes and Implicated in Zinc. Homeostasis Plant Cell Physiol 45:1749-1758. https://doi.org/10.1093/pcp/pci015

20. Leon-Mediavilla J et al. (2018) MtMTP2-Facilitated Zinc Transport Into Intracellular Compartments Is Essential for Nodule Development in Medicago truncatula Front. Plant Sci 9:990. https://doi.org/10.3389/fpls.2018.00990

21. Li H et al. (2019) Identification and expression analysis of the GDSL esterase/lipase family genes, and the characterization of SaGLIP8 in Sedum alfredii Hance under cadmium stress. Peerj 7:e6741. https://doi.org/10.7717/peerj.6741

22. Li X, Wu Y, Li B, He W, Yang Y, Yang Y (2018) Genome-Wide Identification and Expression Analysis of the Cation Diffusion Facilitator Gene Family in Turnip Under Diverse Metal Ion Stresses. Front Genet 9:103. https://doi.org/10.3389/fgene.2018.00103

23. Li Y et al. (2019) Differences of Cd uptake and expression of MT family genes and NRAMP2 in two varieties of ryegrasses. Environ Sci Pollut R 26:13738-13745. https://doi.org/10.1007/s11356-018-2649-z

24. Liu J, Gao Y, Tang Y, Wang D, Chen X, Yao Y, Guo Y (2019) Genome-Wide Identification, Comprehensive Gene Feature, Evolution, and Expression Analysis of Plant Metal Tolerance Proteins in Tobacco Under Heavy Metal Toxicity. Front Genet 10:345. https://doi.org/10.3389/fgene.2019.00345

25. Lu Z et al. (2020) A Single Amino Acid Change in Nramp6 from Sedum Alfredii Hance Affects Cadmium Accumulation. Int J Mol Sci 21:3169. https://doi.org/10.3390/ijms21093169

26. Ma G et al. (2018) OsMTP11, a trans-Golgi network localized transporter, is involved in manganese tolerance in rice. Plant Sci 274:59-69. https://doi.org/ 10.1016/j.plantsci.2018.05.011

27. Migeon A et al. (2010) Genome-wide analysis of plant metal transporters, with an emphasis on poplar. Cell Mol Life Sci 67:3763-3784. https://doi.org/10.1007/s00018-010-0445-0

28. Migocka M, Małas K, Maciaszczyk-Dziubinska E, Posyniak E, Migdal I, Szczech P (2018) Cucumber Golgi protein CsMTP5 forms a Zn-transporting heterodimer with high molecular mass protein CsMTP12. Plant Sci 277:196-206. https://doi.org/10.1016/j.plantsci.2018.09.011

29. Migocka M, Papierniak A, Maciaszczyk-Dziubińska E, Poździk P, Posyniak E, Garbiec A, Filleur S (2014) Cucumber metal transport protein MTP8 confers increased tolerance to manganese when expressed in yeast and Arabidopsis thaliana. J Exp Bot 65:5367-5384. https://doi.org/10.1093/jxb/eru295

30. Montanini B, Blaudez D, Jeandroz S, Sanders D, Chalot M (2007) Phylogenetic and functional analysis of the Cation Diffusion Facilitator (CDF) family: improved signature and prediction of substrate specificity. Bmc Genomics 8:107. https://doi.org/10.1186/1471-2164-8-107

Page $12 / 20$ 
31. Papierniak-Wygladala A, Kozak K, Barabasz A, Palusińska M, Całka M, Maślińska K, Antosiewicz DM (2020) Identification and characterization of a tobacco metal tolerance protein, NtMTP2. Metallomics https://doi.org/10.1039/D0MT00210K

32. Paulsen IT, Saier JMH (1997) A Novel Family of Ubiquitous Heavy Metal Ion Transport Proteins. The Journal of Membrane Biology 156:99-103. https://doi.org/10.1007/s002329900192

33. Ram H, Kaur A, Gandass N, Singh S, Deshmukh R, Sonah H, Sharma TR (2019) Molecular characterization and expression dynamics of MTP genes under various spatio-temporal stages and metal stress conditions in rice. Plos One 14:e217360. https://doi.org/10.1371/journal.pone.0217360

34. Russell D, Soulimane T (2012) Evidence for zinc and cadmium binding in a CDF transporter lacking the cytoplasmic domain. Febs Lett 586:4332-4338. https://doi.org/ 10.1016/j.febslet.2012.10.043

35. Shirazi Z, Abedi A, Kordrostami M, Burritt DJ, Hossain MA (2019) Genome-wide identification and characterization of the metal tolerance protein (MTP) family in grape (Vitis vinifera L.). 3 Biotech 9:199.

https://doi.org/10.1007/s13205-019-1728-2

36. Tian W, He G, Qin L, Li D, Meng L, Huang Y, He T (2021) Genome-wide analysis of the NRAMP gene family in potato (Solanum tuberosum): Identification, expression analysis and response to five heavy metals stress Ecotox Environ Safe. 208:111661. https://doi.org/10.1016/j.ecoenv.2020.111661

37. Tsunemitsu $Y$ et al. (2018) A member of cation diffusion facilitator family, MTP11, is required for manganese tolerance and high fertility in rice. Planta 248:231-241. https://doi.org/10.1007/s00425-018-2890-1

38. Ueno D et al. (2015) A polarly localized transporter for efficient manganese uptake in rice. Nat Plants 1:15170. https://doi.org/10.1038/nplants.2015.170

39. van der Zaal BJ, Neuteboom LW, Pinas JE, Chardonnens AN, Schat H, Verkleij JA, Hooykaas PJ (1999) Overexpression of a novel Arabidopsis gene related to putative zinc-transporter genes from animals can lead to enhanced zinc resistance and accumulation. Plant Physiol 119:1047-1055. https://doi.org/10.1104/pp.119.3.1047

40. Vishwakarma K et al. (2019) Avenues of the membrane transport system in adaptation of plants to abiotic stresses. Crit Rev Biotechnol 39:861-883. https://doi.org/10.1080/07388551.2019.1616669

41. Wang F, Qiao K, Liang S, Tian S, Tian Y, Wang H, Chai T (2018) Triticum urartu MTP1: its ability to maintain Zn²+ and $\mathrm{Co}^{2+}$ homeostasis and metal selectivity determinants. Plant Cell Rep 37:1653-1666. https://doi.org/10.1007/s00299-018-2336-z

42. Wang Y et al. (2012) MCScanX: a toolkit for detection and evolutionary analysis of gene synteny and collinearity. Nucleic Acids Res 40:e49. https://doi.org/10.1093/nar/gkr1293

43. Zhang $X$ et al. (2020) Identification of MTP gene family in tea plant (Camellia sinensis L.) and characterization of CsMTP8.2 in manganese toxicity. Ecotox Environ Safe 202:110904. https://doi.org/ 10.1016/j.ecoenv.2020.110904

44. Zhang Y, Wang Y, Ding Z, Wang H, Song L, Jia S, Ma D (2017) Zinc stress affects ionome and metabolome in tea plants. Plant Physiol Bioch 111:318-328. https://doi.org/ 10.1016/j.plaphy.2016.12.014

\section{Figures}



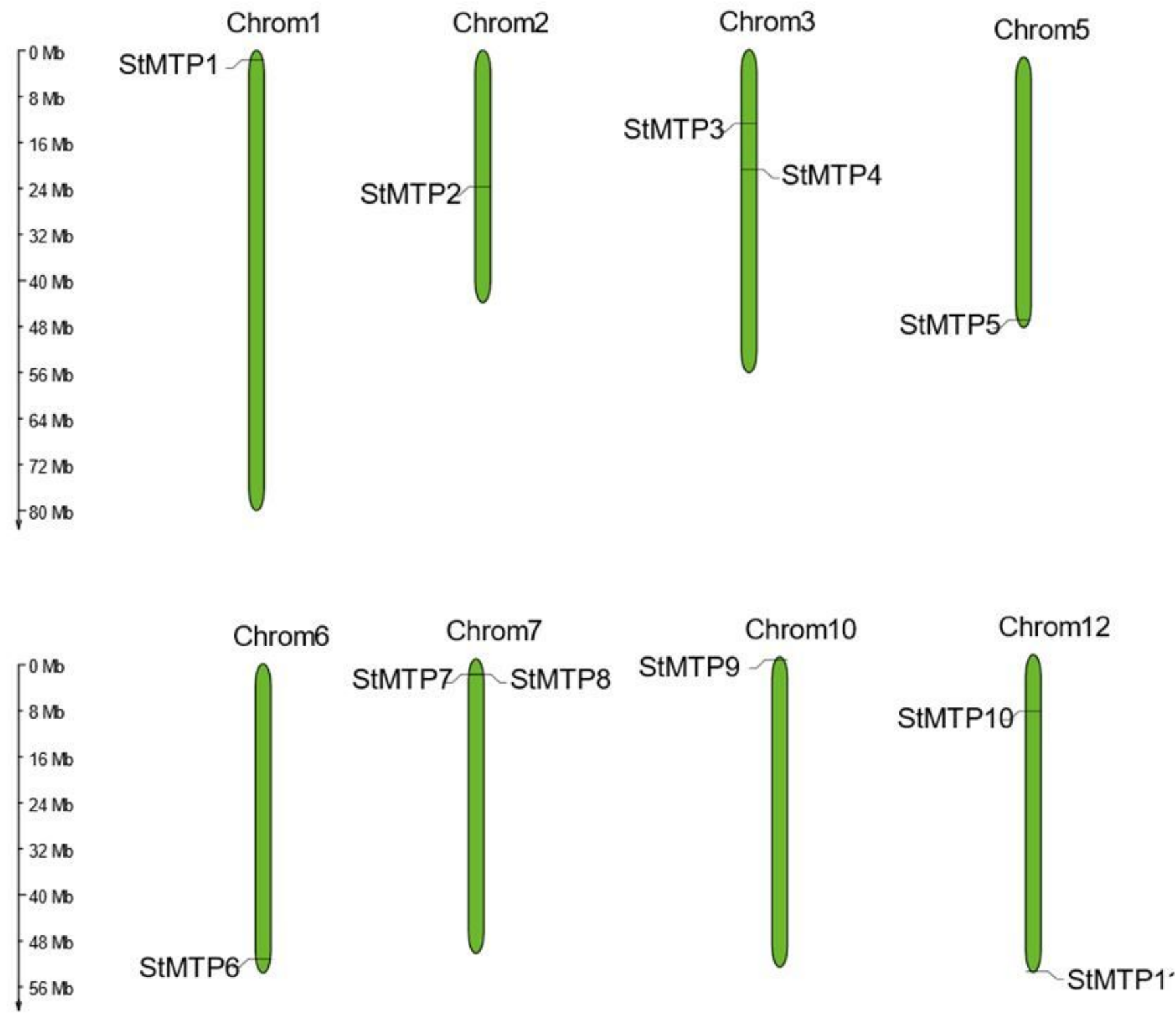

Figure 1

Distributions of StMTP genes on the chromosomes of Solanum tuberosum. The chromosome number is indicated at the top of each chromosome and the size of each chromosome is shown on the left of the figure. 


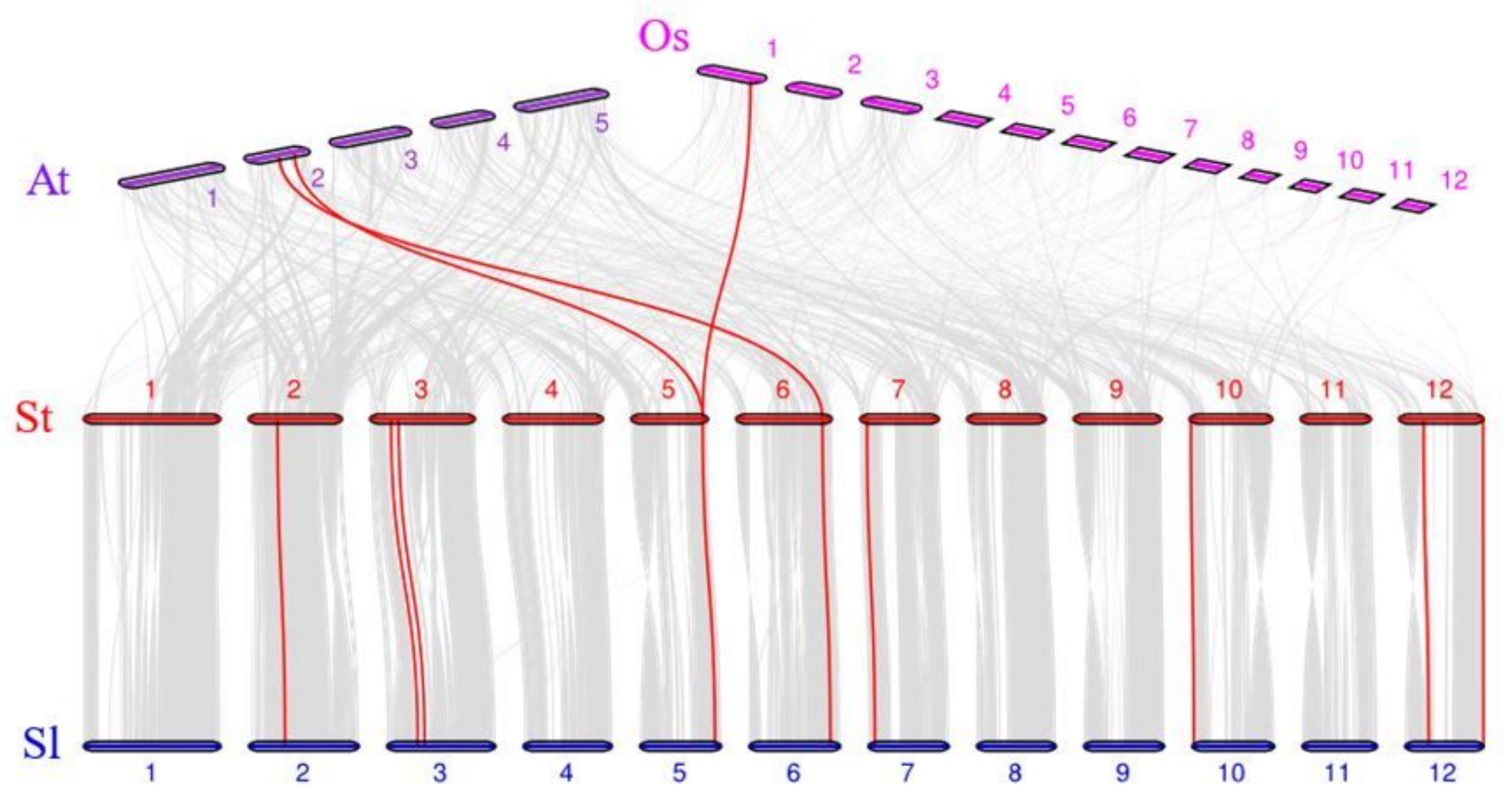

Figure 2

Collinearity analysis of the MTP genes in Solanum tuberosum (St), Solanum lycopersicum (SI), Arabidopsis thaliana (At) and Oryza sativa (Os). The gray lines indicate the collinear block of potato in the genome of the other plants and the red line indicates the synonymous MTP gene pairs. 


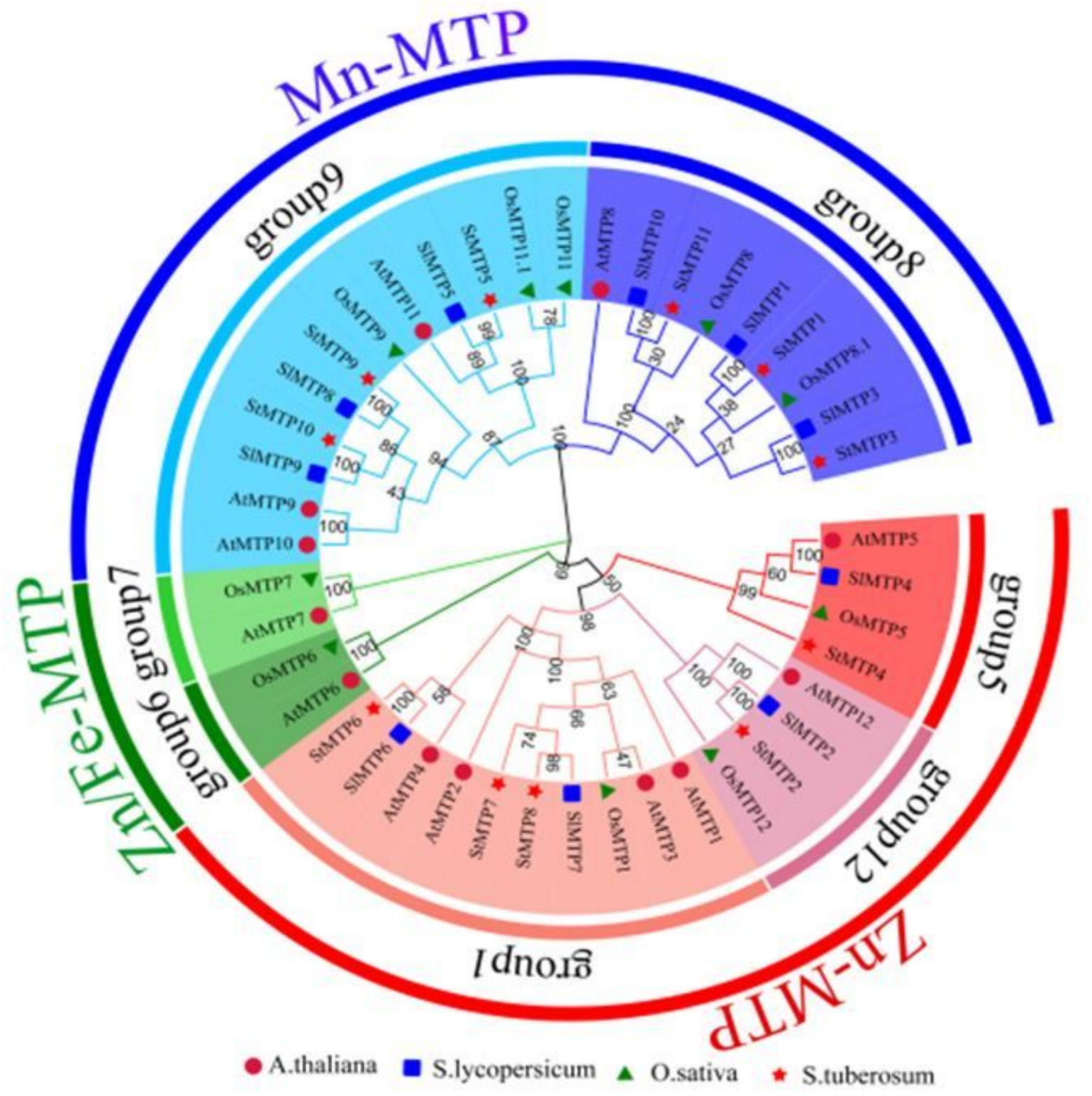

Figure 3

The phylogenic tree was obtained using the neighbor-joining (N-J) method in the MEGA-X (v10.1.8) software. The red five-pointed star represents potatoes, the blue squares represent tomatoes, the red solid circles represent Arabidopsis and the green triangles represent rice. 
(A)

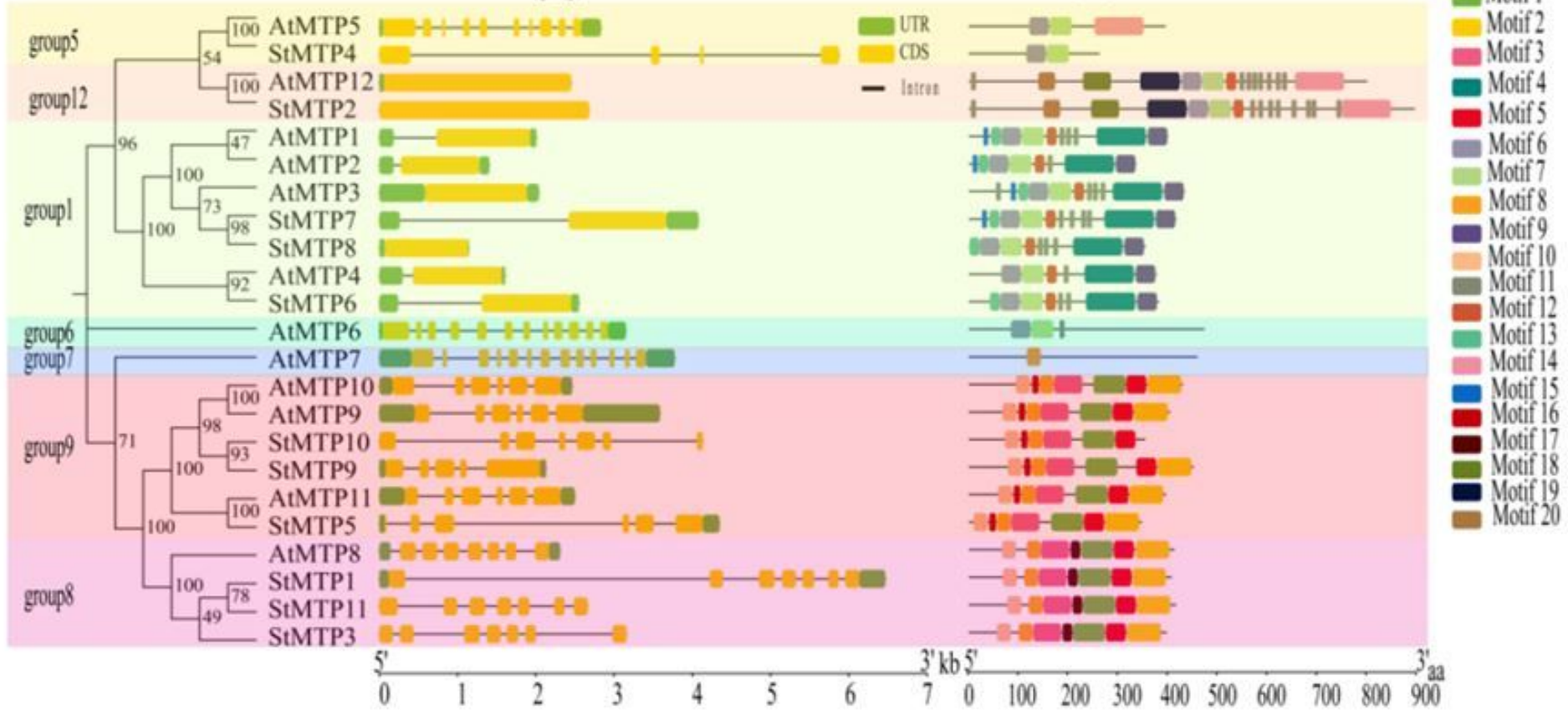

Figure 4

StMTP gene structure analysis and conservative motif analysis. A: The exon-intron structure of StMTP genes, the yellow box represents the untranslated $5^{\prime}$ and $3^{\prime}$ regions of the UTR, the green box represents the exons, the black line represents the introns. The scale bar estimates the length of the exons, introns and untranslated regions. B: the conserved motif. Subject analysis was carried out using the MEME software. Different colored boxes represent the various motifs and the positions in each sequence are marked. The bottom scale bar indicates the length of the MTP protein sequence. 


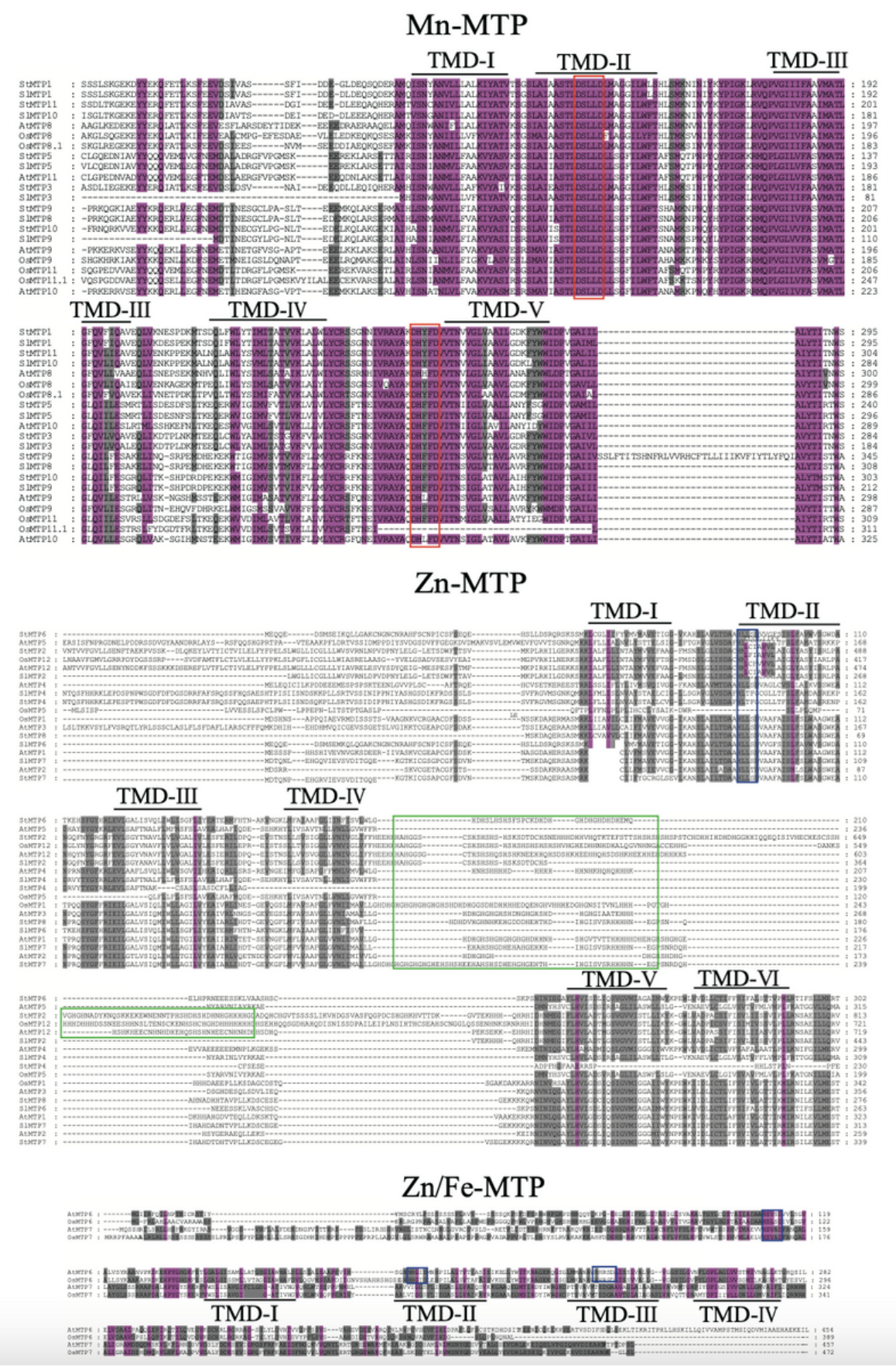

\section{Figure 5}

Sequence analysis of the potato MTP family genes. Gray and pink colors indicate similar amino acid sequences; red indicates similarity $>90 \%$ and gray indicates similarity between $60 \%$ and $90 \%$ ). The transmembrane domains (TMDs) are shown as the lines above these sequences, numbered I to VI. The consensus sequence HXXXD (X = any amino acid) is marked in blue and the DXXXD is marked in red. Zinc-rich areas are shown by green boxes. In the Zn/Fe-MTP subfamily, OsMTP6 and AtMTP6 have no transmembrane domain, so we mark the transmembrane domain below. 


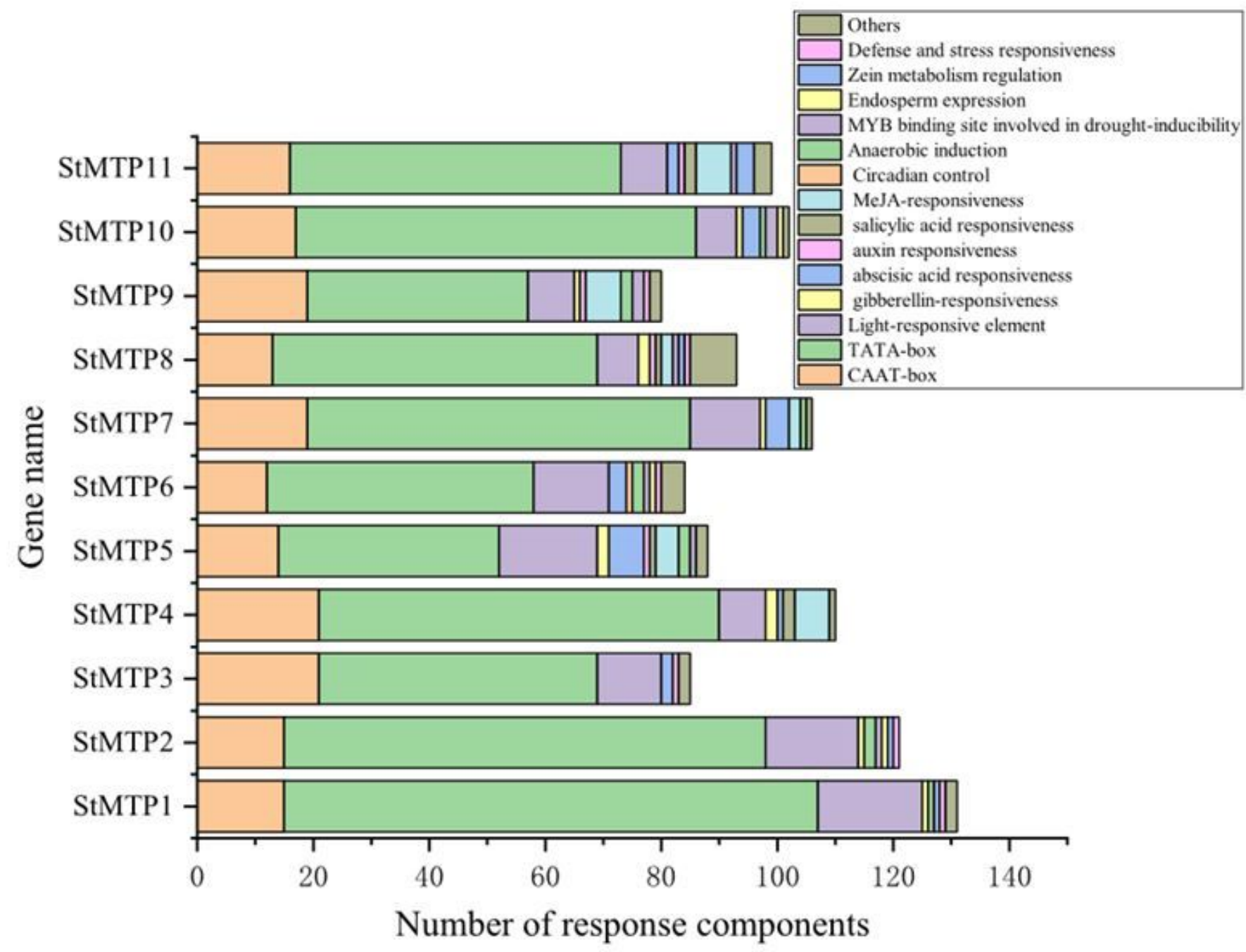

Figure 6

The promoter sequences of the 2,000 bp upstream region of the start codon of the StMTP1-11 genes were analyzed using Plantcare. The abscissa indicates the number of promoters and the ordinate indicates the gene names. 

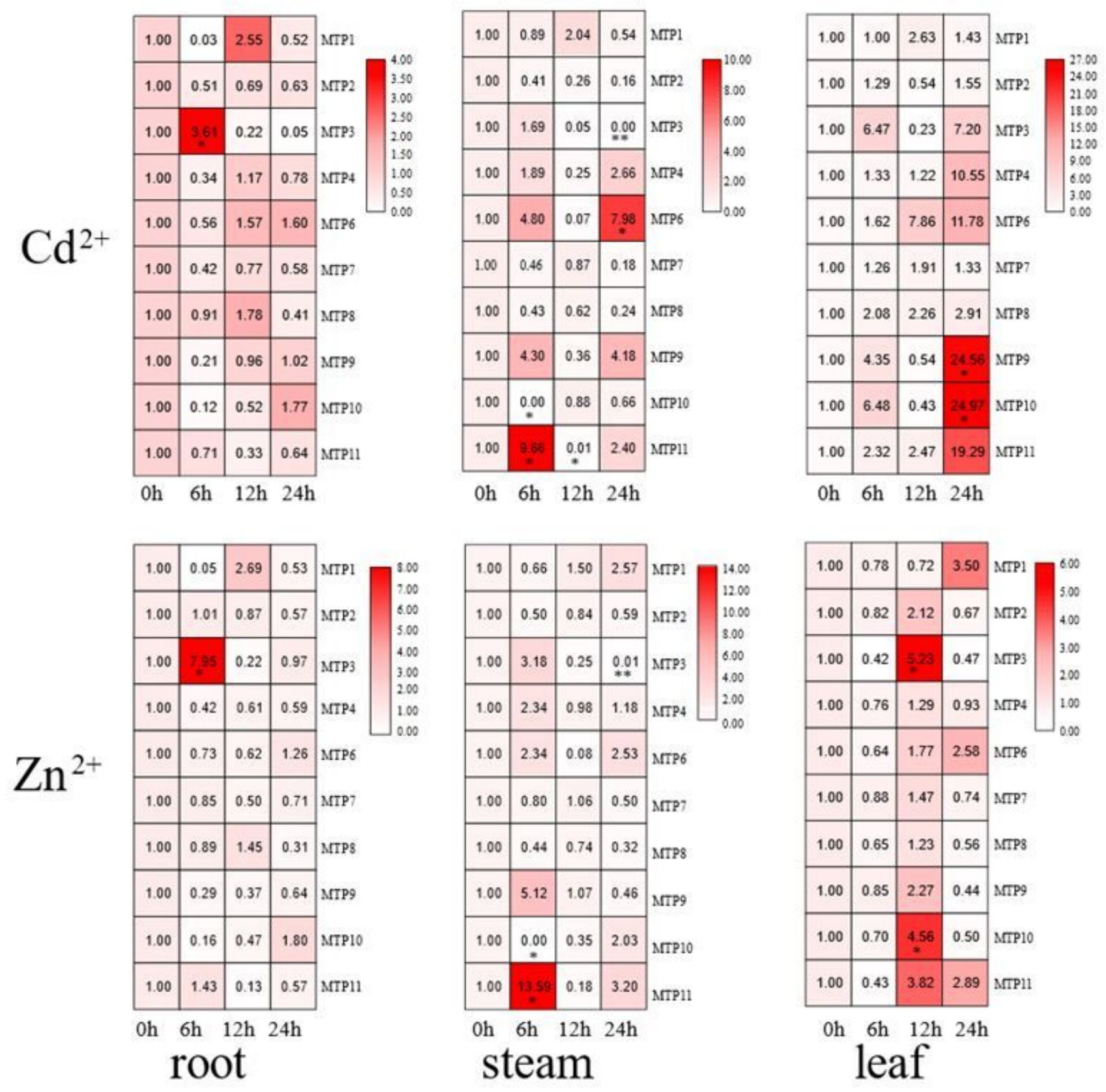

\section{Figure 7}

The expression profile of the Solanum tuberosum MTP genes in different tissues. Total RNA was extracted from the roots, stems and leaves and the expression levels of MTP genes were evaluated by RT-PCR and visualized by TBtools software. The scale bar on the right indicates the relative expression levels. An asterisk indicates a correlation with a significance level of 0.05 (t-test, $\left.{ }^{*} \mathrm{p}<0.05\right)$.

\section{Supplementary Files}

This is a list of supplementary files associated with this preprint. Click to download.

- Supportmaterials.xls 